\title{
X-RAY TOPOGRAPHY USING SYNCHROTRON RADIATION
}

\author{
K. WIETESKA \\ Institute of Atomic Energy, 05-400 Otwock-Świcrk, Poland
}

\begin{abstract}
X-ray diffraction topography is a widely used method to study crystal lattice defects by visualization. The properties of synchrotron radiation relevant to topography methods extend the possibilities of investigations. These properties are the following: a high intensity, a broad spectral range, a natural collimation, a linear polarization in the horizontal plane, and a pulsed time structure. The application of synchrotron radiation to X-ray topographic studies is described and some recent examples of experiments are presented.
\end{abstract}

PACS numbers: $07.85 .+11,61.10 . \mathrm{Lx}$

\section{Introduction}

$\mathrm{X}$-ray topography is an imaging technique based on the difference in reflecting power between distorted areas of the crystal and its perfect parts. A perfect crystal gives a homogeneous image whereas a defect causes a strain field and results in a local intensity change. The other factor determining intensity is simply the local variation of lattice planes orientation of the perfect part of the crystal. These two kinds of contrast are appropriately called extinction and orientation contrast. In such a way X-ray topography reveals dislocations, large precipitations, stacking faults, grains and grain boundaries, domain walls, layer structures, etc.

The principles of reflection X-ray topography were proposed by Berg [1], Barrett [2] and Schulz [3]. In the Berg-Barrett method the characteristic radiation from a linefocus was used and geometrical resolution of a few $\mu \mathrm{m}$ was achieved. It was shown by Newkirk [4] that single dislocations could be seen by this method and their Burgers vector could be determined. Schultz used a white radiation from a point source to study mutual grain misorientation. In the method proposed by Auleytner [5], an X-ray beam from a point focus collimated by a narrow slit hits the surface of the crystal which may oscillate also together with the film. This method is used for the study of mosaic structure, small angle boundaries and dislocation density [6-8]. Guinier and Tennevin [9] used a white radiation divergent beam from a point focus for investigation the real structure of crystals in transmission geometry. 
The most commonly used technique is the Lang method $[10,11]$ of scanning projection and section topography.

The double crystal arrangement [12] is the method where the X-ray beam is reflected from a perfect reference crystal, then from the specimen. Both crystals consist of the same material with the same lattice spacing. This makes the method very sensitive to minute strains and tilts (the local parameter changes of the order of $\left.\Delta d / d=10^{-8}-10^{-9}[13]\right)$.

Another technique is the $\mathrm{X}$-ray moiré topography which is also a very sensitive tool for the study of crystal lattice defects. There are generally two experimental approaches to this method, the Bonse and IIart interferometer [14] cut from a monolithic block of perfect crystal, and the Brádler and Lang method [15] of superposition of separate crystals. The local lattice dilatations and rotations, according to the optical model [16], cause dilatation and rotation moire fringes observed in the topographs. Another important property of X-ray moiré technique is the possibility of the precise measurement of atomic scattering factors and the absolute measurement of lattice parameter [17].

The appearance of high-energy synchrotrons and storage rings emitting electromagnetic radiation with a continuous spectrum that includes a range convenient for diffraction by crystals, caused further rapid development of X-ray diffraction imaging methods. In 1971 synchrotron radiation was used for X-ray diffraction [18] and in 1974 the first X-ray topographs were obtained [19].

\section{Characteristics of synchrotron radiation relevant for X-ray topography}

\subsection{Wavelenglh spread}

The synchrotron radiation has a continuous spectrum [20] with a broad maximum close to the so-called critical wavelength given by

$$
\lambda_{c}=\frac{4 \pi R}{3}\left(\frac{m c^{2}}{E}\right)^{\dot{3}},
$$

where $R$ is the radius of the orbit and $E$ is the kinetic energy of the accelerated particles. The range is usually $0.1-2.5 \AA$ which is very convenient for diffraction experiments. Generally, the interval $0.05-6 \AA$ of one to perform the topography at any selected wavelength.

\subsection{Collimation}

The geometry of synchrotron radiation sources is also very convenient for $\mathrm{X}$-ray topography. The beam is naturally collimated, where typical value of horizontal divergence is about $30^{\prime \prime}$ and vertical one about $0.5^{\prime \prime}$. Because of the small effective focus size and the large source-specimen distance $(20-100 \mathrm{~m})$, the geometrical resolution of the order of $1 \mu \mathrm{m}$ may be obtained at the large areas of the sample. The large specimen-detector distance enables one to use any additional equipment like magnets, heaters, strain devices etc. [21-23]. 


\subsection{Inlensily}

The intensity of synchrotron radiation is $10^{3}-10^{4}$ times higher than that of the best X-ray tubes. Typical exposure times with conventional sources are 1-20 hours. The same experiments done with synchrotron radiation need the exposure time reduced by the corresponding number of orders of magnitude (a few seconds or less). The high intensily facilities permit the use of direct imaging TV detectors [24] and in natural way enable one to perform dynamic experiments step-by-step or in real time. The high intensity allows for the introduction of multi crystal arrangements [25] and interesting optical elements like a plane wave monochromator [26] to form divergence and energy bandwidth of the beam.

\subsection{Polarizalion}

Synchrotron radiation is highly polarized in the electron orbit plane. This property opens a new field of polarized X-ray experiments. By changing the incident plane, one may change the state of polarization of the beam. Also, using horizontal slit moving vertically, thus taking the radiation outside the orbit plane, one obtains elliptically a polarized beam. There are also other ways to obtain circularly polarized X-rays $[27,28]$ needed for instance for magnetic experiments.

\subsection{Pulsed lime structure}

The circulating particles are grouped in so-called bunches. Thus, the synchrotron radiation has a time structure, at typical MIIz rates. The variable parameter determining this time structure is the number of stored bunches whereas the fixed parameters are a length of an individual bunch and its circulation time. As an example, DORIS operates with $150 \mathrm{ps}$ of the length of an individual bunch and $960 \mathrm{~ns}$ of the circulation time of a bunch. The number of stored bunches varies between 1 and 480 . Thus, the pulse repetition time is 2 ns to 960 ns. This time structure permits stroboscopic topography when the exact synchronization of the observed process as the propagation of acoustic waves is realized [29-33]. The contrast observed in stroboscopic surface acoustic wave topography was studied and simulated by ray tracing calculations [31]. The Bragg reflection was approximated by mirror reflection from a wave shaped crystal surface. Because this model is not restricted to X-rays, but holds for visible light as well, the "topographs" of surface acoustic waves were obtained using a visible part of synchrotron radiation [34].

\section{The intensities of the Lane reflections}

$\mathrm{X}$-ray topography with white synchrotron radiation is a very simple and powerful tool for crystal defects studies. In this technique the Bragg law is satisfied as in a conventional Laue photograph. An example of the white beam picture is shown in Fig. 1.

The wide range of wavelengths causes that in Laue patterns all orders from a particular set of planes are superimposed in the same region of photoemulsion. The image is formed from a fundamental wavelength and higher harmonics and, 


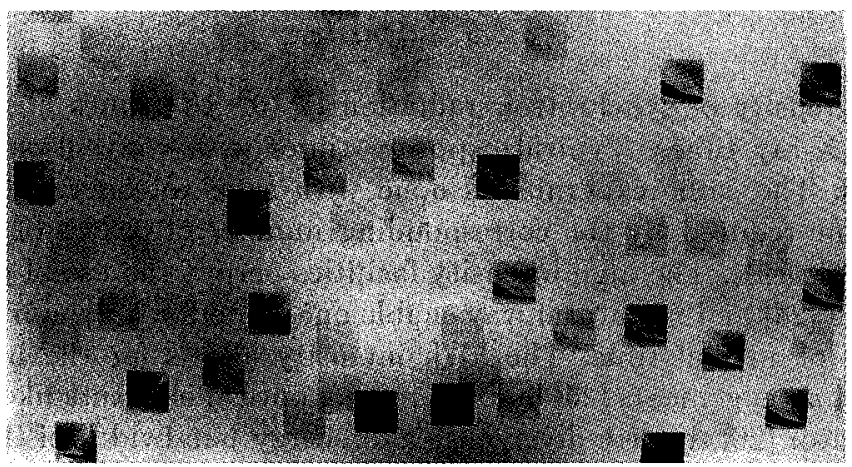

Fig. 1. Laue topographs from a GGG crystal obtained with white synchrotron radiation. The details visible in some of the spots are growth striations and facets (one to one copy of the original film).

as it was pointed out $[19,35]$, the intensity in the topograph is integrated over the wavelengths diffracted by the certain Bragg reflection. The intensity for a given reflection can be written as follows:

$$
I \approx|C| \lambda^{3} p(\lambda) F_{h} \frac{1}{\sin ^{2} 0}\left(\frac{\gamma_{0}}{\gamma_{h}}\right)^{1 / 2} \frac{e^{2}}{m c^{2}} \frac{1}{\pi V},
$$

where $C$ is the polarization factor which is $|\cos 20|$ for horizontal dispersion and 1 for vertical dispersion, $P(\lambda)$ is the percentage spectral cnergy distribution at $\lambda, F_{h}$ is the structure factor, $e^{2} / m c^{2}$ is the classical electron radius, $V$ is the volume of the unit cell and $\gamma_{0}=n \cdot k_{0}, \gamma_{h}=n \cdot k_{h}$ are the direction cosines of the incident and diffracted waves. The basic intensity forming each topograph and recorded by the film is given by

$$
I \approx \sum_{i} P\left(\lambda_{i}\right) F_{h} \lambda_{i}^{3} E_{\mathrm{a}}\left(\lambda_{i}\right) E_{\mathrm{e}}\left(\lambda_{i}\right) B_{i}\left(\lambda_{i}\right)
$$

where $i=1,2,3 \ldots$ represents order of reflection, $E_{\mathrm{a}}$ and $E_{\mathrm{e}}$ are the exponential absorptions of air and crystal and $B_{i}\left(\lambda_{i}\right)$ represents the sensitivity of photoemulsion for different wavelengths. Generally, there is a few ways to eliminate the harmonics, for instance by using a monochromator with successive reflections with either one asymmetric reflection [36] or a slight detuning betwcen two symmetric reflections [37], but in such a case the advantages of the white beam topography and its simplicity are lost. The application of a totally reflecting mirror, to cut off unwanted higher energies would be a very good solution, but the quality of the plane mirror used in a first attempt was not sufficient and horizontal fringes on the topographs were seen. The kind of compromise between monochromatic and white beam topography is the use of an oscillating monolithic monochromator crystal [38].

\section{Examples}

One of the important advantage of white beam topography with synchrotron radiation is the imaging of the sample of poor quality and a polycrystalline sample 
a

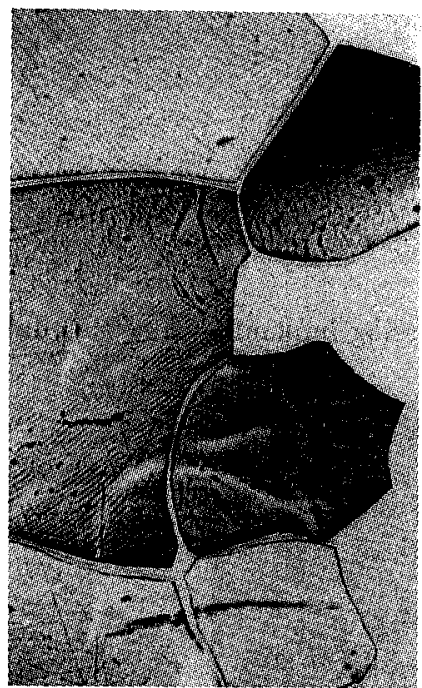

$\mathrm{b}$

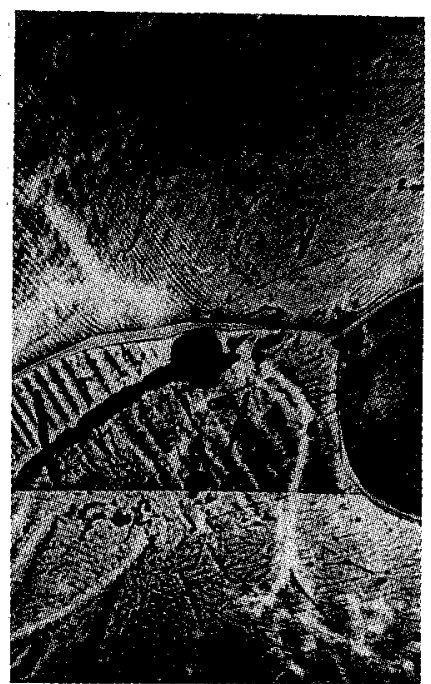

Fig. 2. Reconstructed back reflection topographs of (100) oriented Fe-3\%Si polycrystal showing magnetic domain pattern. (a) All grains are (100) oriented. (b) The grain with a distinct black defect is (111) oriented whereas the neighbouring grains have (100) orientation.

a

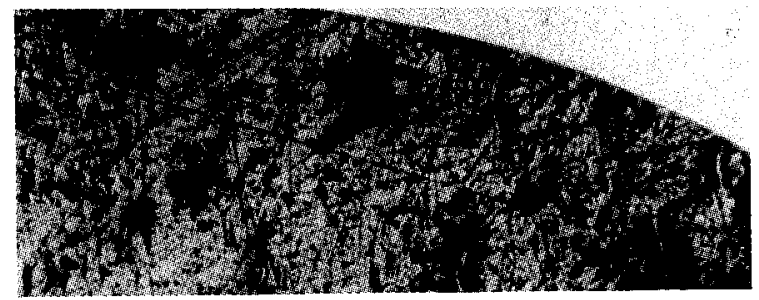

b.

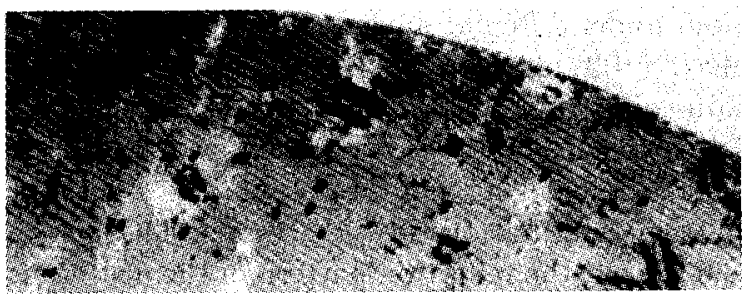

Fig. 3. Transmission topograph of Fe-Si crystal: (a) (200) reflection, (b) (020) reflection.

with a number of grains. Because each grain of any orientation selects the appropriate wavelength from the beam, the reconstructed topograph of whole crystal surface may be taken during one exposition. The examples are shown in Fig. 2.

A large number of spots on the photograph enables one to observe the defect 


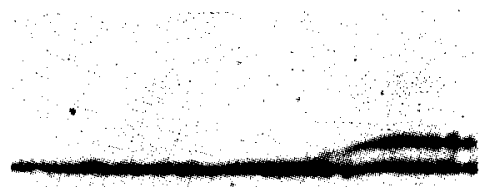

Fig. 4. Reflection image taken with the slit crossing inplanted area boundary.

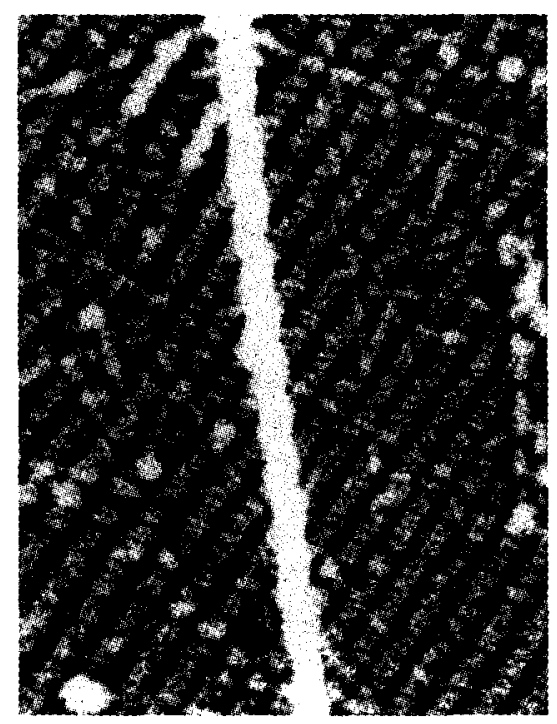

Fig. 5. Reflection topograph of Fe-Si taken with the $50 \mu \mathrm{m}$ wire placed in front of the crystal.

contrast behaviour under different diffraction conditions. Figure 3 shows (200) and (020) topographs of (100) oriented Fe-Si crystal taken from one film. A disappearing of the domain contrasts is seen in (200) reflection.

As it was mentioned above, the experiments with synchrotron radiation have a very convenient geometry. In many cases it makes possible to reveal directly some kinds of lattice deformations in the crystals. The first example is shown in Fig. 4. Using a $30 \mu \mathrm{m}$ slit the reflection pattern of the deuterons implanted gallium arsenide was done. The additional line caused by the shot-through crystal layer with larger lattice plane spacing is noticeable. From geometrical considerations it is possible to determine the value of the lattice parameter change as well as the mutual misorientation of the layers. This method is not as sensitive as double crystal measurements but is very quick and an accuracy of about $10^{-4}$ in relative lattice parameter change may be achieved.

The second example is shown in Fig. 5. In a (001) oriented Fe-Si crystal one $180^{\circ}$ wall and two $90^{\circ}$ walls form an $Y$ shape. The $90^{\circ}$ walls from neighbouring 


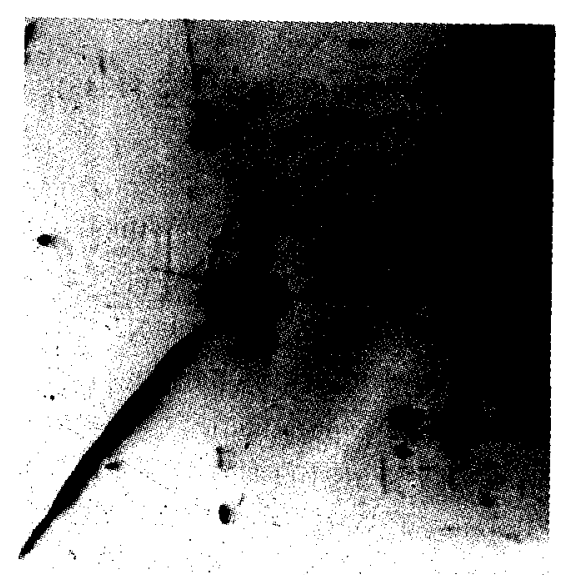

Fig. 6. Unknown defect revealed inside the $10 \mathrm{~mm}$ thick Si crystal. Transmission topograph taken with $1 \mathrm{~s}$ exposure time.

junctions intersect the surface. In one domain, the (001) planes are concavely bent around the [100] axis. Thus, the magnetostriction causes the crests on the top of main domains with magnetization perpendicular to the surface and respectively the troughs between them. $\Lambda$ wire of $50 \mu \mathrm{m}$ diameter was placed in front of the crystal and reflection topograph was taken. The periodic focusing is seen which indicates wave the shape perturbed crystal surface.

Because of the ligh intensity of synchrotron radiation, the much thicker samples can be studied in transmission geometry than when using conventional sources. For example (Fig. 6), the exposure time of a $10 \mathrm{~mm}$ thick silicon crystal was $1 \mathrm{~s}$ with a principal wavelength $\lambda=0.2 \AA$. The topograph revealed an unknown defect inside the crystal.

\section{Ackuowledgments}

The synchrotron radiation experiments were done at IIASYLAB. Financial support by DESY and BMFT project number 05305AXBO is gratefully acknowledged.

\section{References}

[1] W.F. Berg, Nalurwissenschafien 10, 391 (1931).

[2] G.S. Barret, Trans. AIME 161, 15 (1945).

[3] L.G. Schulz, Trans. AIME 200, 1082 (1954).

[4] J.B. Newkirk, Trans. AINE 215, 483 (1959).

[5] J. Aulcytner, Acta Phys. Pol. 17, 111 (1958).

[6] J. Auleytner, Acta Phys. Pol. 18, 81 (1959).

[7] J. Auleytner, Festkörperphysik, Akademie-Verlag, Berlin 1961, p. 134. 
[8] T. Bedyńska, Acta Phys. Pol. 19, 443 (1960).

[9] A. Guinier, J. Tennevin, Acta Crystallogr. 2, 133 (1949).

[10] A.R. Lang, J. Appl. Phys. 29, 597 (1958).

[11] A.R. Lang, Acta Crystallogr. 12, 249 (1959).

[12] U. Bonse, E. Kappler, Z. Nat.forsch. A 13, 348 (1958).

[13] U. Bonse, J. Hartmann, Z. Kristallogr. 156, 265 (1981).

[14] U. Bonse, M. Hart, Appl. Phys. Lelt. 6, 155 (1965).

[15] J. Brádler, A.R. Lang, Acta Crystallogr. A 24, 246 (1968).

[16] U. Bonse, M. Hart. Z: Phys. 190, 455 (1965).

[17] U. Bonse, M. Hart. Z. Phys. 188, 154 (1965).

[18] G. Rosenbaum, K.C. Holmes, J. Witz, Nalure 230, 434 (1971).

[19] T. Tuomi, K. Naukkarinen, P. Rabe, Phys. Status Solidi A 25, 93 (1974).

[20] R.P. Godwin, Springer Tracts Mod. Phys. 51, 1 (1969).

[21] B.K. Tanner, O. Midgley, M. Safa, J. Appl. Cryst. 10, 281 (1977).

[22] J. Gastaldi, C. Jourdan, P. Marzo, C. Allasia, J.V. Jullien, J. Appl. Crystallogr. 15, 391 (1982).

[23] O.P. Aleshko-Ozhevskij, Cristallographiya 27, 1125 (1982).

[24] J.K. Cringean, B.K. Tanner, Progr. Cryst. Growth Charact. 14, 403 (1987).

[25] W.J. Boettinger, R.C. Dobbyn, H.E. Burdette, M. Kuriyama, Nucl. Instrum. Methods 105, 355 (1982).

[26] M. Souvage, C. Malgrange, J.F. Petroff, J. Appl. Crystallogr. 16, 14 (1983).

[27] P. Elleaume, ESRF Report SR/1D 8823; Nucl. Instrum. Methods Phys. Res. A 291, 371 (1990).

[28] C. Malgrange, C. Carvalho, L. Braicovinch, J. Goulon, Nucl. Instrum. Methods Phys. Res. A 308, 390 (1991).

[29] R.W. Whatmore, P.A. Goddard, B.K. Tanner, G.F. Clark, Nature 299, 44 (1982).

[30] R.W. Whatmore, P.A. Goddard, B.K. Tanner, IEEE Ultrasonic Symp. Proc., Vol. 1, San Diego 1982, p. 363.

[31] H. Cerva, W. Graeff, Phys. Status Solidi A 82, 35 (1984).

[32] H. Cerva, W. Graeff, Phys. Status Solidi A 87, 507 (1985).

[33] H. Cerva, W. Graeff, Pliys. Status Solidi A 93, K129 (1986).

[34] W. Gaeff, K. Wieteska, Nukleonika, in print.

[35] M. Hart, J. Appl. Crystallogr. 8, 436 (1975).

[36] T. Matsushita, H. Hashizume, in: Handbook on Synchrotron Radiation, Ed.

E.E. Koch, Vol. 1, North-Holland, Amsterdam 1983, p. 261.

[37] M. Hart, A.R.D. Rodrigues, J. Appl. Crystallogr. 11, 948 (1978).

[38] W. Graeff, K. Wieteska, J. Sci. Techn. 3, 152 (1992). 